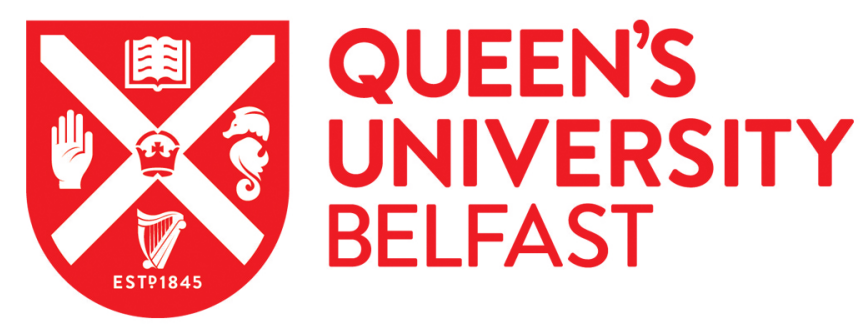

\title{
Identification of synergists that potentiate the action of polymyxin B against Burkholderia cenocepacia
}

Loutet, S. A., El-Halfawy, O. M., Jassem, A. N., Sánchez López, J. M., Fernández Medarde, A., Speert, D. P., Davies, J. E., \& Valvano, M. A. (2015). Identification of synergists that potentiate the action of polymyxin B against Burkholderia cenocepacia. International Journal of Antimicrobial Agents, 46(4), 376-380. https://doi.org/10.1016/j.ijantimicag.2015.05.010

Published in:

International Journal of Antimicrobial Agents

Document Version:

Peer reviewed version

Queen's University Belfast - Research Portal:

Link to publication record in Queen's University Belfast Research Portal

Publisher rights

(C) 2015, Elsevier. Licensed under the Creative Commons Attribution -NonCommercial-NoDerivs License

(https://creativecommons.org/licenses/by-nc-nd/4.0/), which permits distribution and reproduction for non-commercial purposes, provided the author and source are cited.

\section{General rights}

Copyright for the publications made accessible via the Queen's University Belfast Research Portal is retained by the author(s) and / or other copyright owners and it is a condition of accessing these publications that users recognise and abide by the legal requirements associated with these rights.

Take down policy

The Research Portal is Queen's institutional repository that provides access to Queen's research output. Every effort has been made to ensure that content in the Research Portal does not infringe any person's rights, or applicable UK laws. If you discover content in the Research Portal that you believe breaches copyright or violates any law, please contact openaccess@qub.ac.uk. 
Identification of synergists that potentiate the action of polymyxin B against Burkholderia cenocepacia

\author{
Slade A. Loutet ${ }^{1,4 \dagger}$, Omar M. El-Halfawy ${ }^{1,2 \dagger}$, Agatha N. Jassem ${ }^{5}$, José María Sánchez \\ López $^{8}$, Antonio Fernández Medarde ${ }^{8}$, David P. Speert ${ }^{6,7}$, Julian E. Davies ${ }^{4}$, and Miguel A. \\ Valvano $^{1,3^{*}}$
}

${ }^{1}$ Centre for Human Immunology and Department of Microbiology and Immunology, University of Western Ontario, London, Ontario, Canada. ${ }^{2}$ Department of Pharmaceutical Microbiology, Faculty of Pharmacy, Alexandria University, Egypt. ${ }^{3}$ Centre for Infection and Immunity, Queen's University Belfast, United Kingdom.Departments of ${ }^{4}$ Microbiology and Immunology, ${ }^{5}$ Pathology and Laboratory Medicine, ${ }^{6}$ Pediatrics, and ${ }^{7}$ Pathology, University of British Columbia, Vancouver, BC, Canada. ${ }^{8}$ Biomar Microbial Technologies, León, Spain.

$\dagger$ Authors contributed equally.

*Corresponding Author: Miguel A. Valvano. E-mail: m.valvano@qub.ac.uk

RUNNING TITLE: Synergizers of polymyxins against Burkholderia cenocepacia

\begin{abstract}
Burkholderia cenocepacia and other members of the Burkholderia cepacia complex (Bcc) are highly multidrug-resistant bacteria that cause severe pulmonary infections in patients with cystic fibrosis. A screen of 2686 compounds derived from marine organisms identified molecules that could synergize with polymyxin B to inhibit growth of $B$. cenocepacia. At $1 \mu \mathrm{g} / \mathrm{ml}$, five compounds synergized with polymyxin B and inhibited the growth of B. cenocepacia by more than $70 \%$ compared to growth in polymyxin B alone. Follow-up testing revealed that one compound from the screen, the aminocoumarin antibiotic novobiocin, synergized with polymyxin $\mathrm{B}$ and colistin against tobramycin-resistant clinical isolates of $B$. cenocepacia and Burkholderia multivorans. In parallel, we show that novobiocin sensitivity is common among Bcc species and these bacteria are even more susceptible to an alternative aminocoumarin, clorobiocin, which also had an additive effect with polymyxin B against $B$. cenocepacia. These studies support using aminocoumarin antibiotics to treat Bcc infections and show that synergizers can be found to increase the efficacy of antimicrobial peptides and polymyxins against Bcc bacteria.
\end{abstract}

\title{
Keywords
}

Burkholderia cepacia complex, polymyxins, novobiocin, clorobiocin, aminocoumarins, cystic fibrosis, antimicrobial adjuvants

\section{Abbreviations}

Bcc, Burkholderia cepacia complex; PmB, polymyxin B; CF, cystic fibrosis; LB, Luria broth; $\mathrm{MIC}$, minimum inhibitory concentration; FIC, fractional inhibitory concentration 


\section{Introduction}

The alarming increase in multidrug resistance [1], particularly intrinsic, high-level resistance, undermines new treatments improving health and extending the life of patients with chronic conditions. For example, respiratory failure secondary to chronic pulmonary bacterial infection in patients with cystic fibrosis (CF) hinders the improvements in survival achieved over the last several decades and remains the primary cause of death [2]. The emergence of a growing number of CF pathogens with intrinsic, multidrug resistance such as Burkholderia cepacia complex (Bcc) bacteria, Stenotrophomonas maltophilia, Achromobacter xylosoxidans, and nontuberculous mycobacteria creates a pressing need for novel therapies [2]. The growing number of multidrug resistant bacteria and the lack of new antibiotics to combat them have led to the revival of the polymyxins, a family of lipid-linked antimicrobial peptides, as a last resort treatment despite their previously reported systemic toxicity [3]. Unfortunately, Bcc bacteria are also resistant to these compounds $[3,4]$. We investigated therapeutic strategies to disrupt the high-level, intrinsic, multidrug resistance of B. cepacia complex, using Burkholderia cenocepacia as a model bacterium. B. cenocepacia is an environmental, opportunistic pathogen that belongs to the Bcc and causes serious respiratory infections in CF patients [5]. These infections are associated with faster decline in lung function, debilitating exacerbations and ultimately death [6], and they also reduce the survival of CF patients after lung transplant [7].

Antimicrobial adjuvants are molecules that inhibit a bacterial resistance mechanism to an antibiotic, effectively increasing the bacterial susceptibility to that antibiotic [8]. This concept has been investigated as an appealing approach to reverse resistance of bacteria to antibiotics [9]. Here, we used a strategy to identify compounds that synergize with polymyxin $\mathrm{B}$ against $B$. cenocepacia by screening a library of naturally occurring molecules derived from marine sources. These synergists reduce the extreme resistance of B. cenocepacia and other Bcc bacteria towards polymyxin $\mathrm{B}(\mathrm{PmB})$ and colistin (polymyxin $\mathrm{E})$, thereby increasing their antimicrobial effectiveness. Clinically, this could provide an effective way to reduce the dosage of polymyxins and therefore increase their efficacy while at the same time overcoming their toxic effects.

\section{Materials and Methods}

\subsection{Bacterial strains.}

B. cenocepacia K56-2 was used in the initial screen; this isolate is from a CF patient and is commonly used as prototypic strain from the $B$. cenocepacia ET-12 epidemic clonal lineage. Follow-up studies were conducted using the previously described Bcc strain panel [10], as well as two tobramycin-resistant B. cenocepacia CF isolates (GIB-C1141144 and GRI-C1210932).

\subsection{Screening a library of compounds for synergism with PmB.}

The library consisted of 2,686 compounds derived from sea life, isolated and characterized by Biomar Microbial Technologies, Spain. Solutions of these compounds were seeded in pairs of 100-well Bioscreen C plates and the solvent was evaporated. Overnight cultures of K56-2 were diluted to an $\mathrm{OD}_{600}$ of 0.0002 in fresh LB medium and added to the plates (300 $\mu \mathrm{l} / \mathrm{well})$, dissolving the compounds to a final concentration of $1 \mu \mathrm{g} / \mathrm{ml}$. Plate pairs were incubated in the Bioscreen $\mathrm{C}$ automated growth curve analyzer at $37^{\circ} \mathrm{C}$ with continuous medium shaking. After $2 \mathrm{~h}, \mathrm{PmB}$ was added to one plate at a final concentration of $500 \mu \mathrm{g} / \mathrm{ml}$ and the vehicle control in which PmB is dissolved $(0.2 \%$ bovine serum albumin, $0.01 \%$ acetic acid) was added to the other plate. This concentration of PmB has little impact on the growth of $B$. 
cenocepacia K56-2 (data not shown). Wells with no compounds were also included in each plate of the pairs. The plates were further incubated at $37^{\circ} \mathrm{C}$ and the $\mathrm{OD}_{600}$ was read every $30 \mathrm{~min}$. Further, one well on each plate had no compound and received sterile LB plus the vehicle control or PmB to provide a negative control and confirm that each experiment was performed aseptically. This experimental design allows the identification of compounds that have direct antimicrobial activity against $B$. cenocepacia as well as those that synergize with PmB.

\subsection{Minimum inhibitory concentration MIC and checkerboard assays.}

MIC assays were conducted as previously described [11]. The incubation time for GIBC1141144 and GRI-C1210932 was extended to $48 \mathrm{~h}$, as these strains grow much slower than K56-2. Checkerboard assays were conducted with combinations of antibiotics. When the MIC values could be determined (novobiocin, clorobiocin, and tobramycin) increasing concentrations in two-fold increments between $1 / 32$ of the MIC value and the MIC value were used. For example, if the novobiocin MIC of a particular strain is $16 \mu \mathrm{g} / \mathrm{ml}, 0.5,1,2,4,8$, and $16 \mu \mathrm{g} / \mathrm{ml}$ were tested in the checkerboard assay. Accurate polymyxin B and colistin MIC values against Bcc bacteria cannot be determined because these bacteria grow at concentrations greater than the solubility of these compounds in growth medium. All Bcc strains grew in $1024 \mu \mathrm{g} / \mathrm{ml}$ polymyxin $\mathrm{B}$ and colistin, the highest concentrations tested. These were considered to be half the MIC value for all of the Bcc strains examined. In checkerboard assays, polymyxin B and colistin were tested at concentrations of 64 (1/32 MIC), 128 (1/16 MIC), 256 (1/8 MIC), 512 (1/4 MIC), and 1024 $\mu \mathrm{g} / \mathrm{ml}(1 / 2 \mathrm{MIC})$. Fractional inhibitory concentration (FIC) indices were calculated as FIC = $\mathrm{A} / \mathrm{MIC}_{\mathrm{A}}+\mathrm{B} / \mathrm{MIC}_{\mathrm{B}}$ where $\mathrm{A}$ and $\mathrm{B}$ are the concentrations of two antibiotics required in combination to inhibit the growth of the bacteria and $\mathrm{MIC}_{\mathrm{A}}$ and $\mathrm{MIC}_{\mathrm{B}}$ are the MIC values for drugs $\mathrm{A}$ and $\mathrm{B}$ alone [12]. An FIC index $\leq 0.5$ was considered synergistic. For synergy studies, all antibiotics came from independent suppliers: novobiocin ( $>90 \%$ pure), polymyxin B ( $>60 \%$ pure), colistin ( $>50 \%$ pure), and tobramycin ( $>98 \%$ pure) were purchased from Sigma. RhonePoulenc Santé donated commercial-grade clorobiocin. Novobiocin and clorobiocin MIC values for the experimental Bcc strain panel were also determined using a conventional broth dilution approach [13]. MIC and checkerboard experiments were independently repeated up to 4 times. In cases where multiple values were obtained between replicates, we reported the larger (more conservative) value.

\section{Results}

\subsection{Small molecule screening for polymyxin B synergizers}

None of the 2686 compounds from marine sources had significant inhibitory effects on the bacterial cells at the concentration tested $(1 \mu \mathrm{g} / \mathrm{ml})$ in the absence of PmB (Fig 1). The highest inhibition in the absence of $\mathrm{PmB}$ was $22.8 \%$. Under these conditions, the hit rate for this library of compounds that directly inhibit B. cenocepacia strain K56-2 is 0\% (0/2686). This does not preclude that they may possess direct antibiotic activity at higher concentrations. As described below, one of the compounds in the library was novobiocin and if the concentration of the library had been $20 \mu \mathrm{g} / \mathrm{ml}$, bacterial growth inhibition in the absence of PmB would have been observed.

The screen revealed five compounds that were effective in potentiating the antimicrobial activity of PmB against B. cenocepacia K56-2 (Fig. 1). The combination of each of them with $\mathrm{PmB}$ inhibited growth by $70 \%$ or more relative to growth in $\mathrm{PmB}$ alone at $20 \mathrm{~h}$. The hit rate for 
compounds that synergize with PmB was $1.9 \%$ (5/2686). These compounds are thielavin B (Fig. 2A), micacocidin B (Fig. 2B), IKD-8344 (Fig. 2C), a novel compound CL0231 (Fig. 2D), and novobiocin (Fig. 2F). Three other compounds with structures similar to micacocidin B but not in complexes with metal were present in the library, but none of them had any synergistic effects with PmB against $B$. cenocepacia. This provides an internal control indicating that the metal in micacocidin $\mathrm{B}$ is required for its activity. A compound similar to CL0231 (CL0236, Fig. 2E) with a phenylalanine replacing a valine residue did not have any synergistic effects with PmB, suggesting that the bioactivity of CL0231 and related compounds depends on their amino acid sequences. Of these five compounds we confirmed the activity in follow-up studies for novobiocin and IKD-8344 (confirmation rate of 40\%, 2/5), as we could not obtain sufficient amounts of the other three compounds to repeat the assays.

\subsection{Novobiocin and polymyxin B synergize against other Burkholderia isolates}

We evaluated whether the synergistic effect of novobiocin against $B$. cenocepacia K56-2 also occurs in other clinical Bcc isolates (Burkholderia multivorans strain C0514 and two tobramycin-resistant Bcc isolates, GIB-C1141144 and GRI-C1210932). In addition to testing $\mathrm{PmB}$, we included colistin and tobramycin in these studies since nebulized formulations of these antibiotics are used to treat pulmonary bacterial infections in CF patients [2]. We first determined the individual MIC values against these Bcc strains. All of them had novobiocin MIC values of $16 \mu \mathrm{g} / \mathrm{ml}$, which were considerably lower than values for Pseudomonas aeruginosa PAO1 $(512 \mu \mathrm{g} / \mathrm{ml})$ and Escherichia coli DH5 $(128 \mu \mathrm{g} / \mathrm{ml})$. Bcc bacteria grew at the highest concentrations tested $(1024 \mu \mathrm{g} / \mathrm{ml})$ for PmB and colistin. Finally, Bcc isolates GIB-C1141144 and GRI-C1210932 were considerably more resistant to tobramycin (MIC=2048 and $4096 \mu \mathrm{g} / \mathrm{ml}$ respectively) than either $\mathrm{K} 56-2$ or $\mathrm{C} 0514(\mathrm{MIC}=256 \mu \mathrm{g} / \mathrm{ml})$.

Checkerboard assays were performed to quantitate levels of synergism. Since the MIC values for all Bcc bacteria with PmB and colistin are greater than the highest concentration tested $(1024 \mu \mathrm{g} / \mathrm{ml})$, we empirically considered $1024 \mu \mathrm{g} / \mathrm{ml}$ to be one half the MIC value. Novobiocin acted synergistically with both $\mathrm{PmB}$ and colistin against the tested Bcc strains (Table 2). Synergism between novobiocin and antimicrobial peptides against Gram-negative bacteria has been previously reported [12]. Novobiocin slightly enhanced the activity of tobramycin against K56-2 and C0514, but the FIC indices were above 0.5 (Table 2). In contrast, sub-MIC concentrations of tobramycin antagonized the activity of novobiocin against the tobramycinresistant isolates GIB-C1141144 and GRI-C1210932 (FIC indices > 1). We speculate that subMIC concentrations of tobramycin activate a mechanism in these strains that provides crossresistance against novobiocin.

Similar experiments with other synergistic compounds detected from the initial screen were not possible due to our inability at this point to extract higher concentrations of these compounds from their marine sources and lack of alternative sources for most of the compounds. Fermentation of these compounds from their producer organisms, their overproduction from a recombinant expression system, or their chemical synthesis are possible options for further evaluation of these compounds in the future.

\subsection{Clorobiocin is more potent than novobiocin against Burkholderia bacteria and also enhances polymyxin $B$ activity}

Given the synergism of novobiocin with polymyxin B, and the low MIC of novobiocin for K56-2, we investigated another aminocoumarin, clorobiocin (Fig. 2G). Clorobiocin was more 
potent than novobiocin; its MIC against K56-2 was $1 \mu \mathrm{g} / \mathrm{ml}$. A parallel experiment comparing the MIC values of the two aminocoumarins against the 30 strains of the Bcc panel [10] also showed that MIC values for clorobiocin were significantly lower than those for novobiocin (Table 2). The FIC value for clorobiocin and PmB against K56-2 was 0.515, indicating that these compounds have an additive effect and are close to the cut-off for synergism.

\section{Discussion}

There is great need for more effective antibiotics against non-fermenting opportunistic Gram-negative bacteria [2]. While polymyxins or other antimicrobial peptides represent an excellent choice, their high dosage to overcome high-level resistance in some of these bacterial species could be problematic due to toxicity and solubility of the peptides in biological solvents. The combination of polymyxins with synergistic molecules that can potentiate their antibacterial activity offers an opportunity for more effective treatments at lower dosages, which will carry a significant benefit to patients by reducing toxicity. In this work, we employed a screening strategy allowing us to detect compounds that synergize with PmB. Five such compounds were found in a library of 2686 compounds obtained from marine sources. This relatively high success ratio provides proof of concept that it is possible to find compounds that could synergize with antimicrobial peptides, reversing the resistance of Bcc bacteria against these last resort antibiotics. As an alternative to screening for antibiotic synergism, development of inhibitors targeting specific pathways involved in Bcc antibiotic resistance might also be an effective strategy for combating Bcc infection. In that context, efforts $[23,24]$ have been made to develop novel therapeutics that specifically target the synthesis pathways of two different components of the lipopolysaccharide molecule, both of which have been implicated in $\mathrm{B}$. cenocepacia antimicrobial peptide resistance $[25,26]$. Furthermore, recent observations point to alternative strategies inhibiting secreted molecules that communicate antibiotic resistance to other less resistant members of a bacterial population [27].

Four compounds (thielavin B, micacocidin B, IKD-8344, and CL0231) identified in the initial screen require further testing before firm conclusions about their synergy with PmB or other compounds can be made. Thielavin B (Fig. 2A) is a prostaglandin synthesis inhibitor produced by the fungus Thielavia terricola [14], that also interferes with peptidoglycan synthesis in Enterococcus faecalis [15]. Micacocidin B (Fig. 2B) is a copper-containing compound produced by Pseudomonas sp. with potent activity against various Mycoplasma species [16]. IKD-8344 (Fig. 2C) is a macrodiolide previously shown to be cytotoxic and anthelmintic [17]. The fourth compound was a novel cyclic peptide coded CL0231 (Fig.2 D).

The fifth compound, novobiocin (Fig. 2F) is a bacteriostatic aminocoumarin antibiotic that binds to the B subunit of DNA gyrase (GyrB) blocking the ATPase activity and hence inhibiting DNA supercoiling [18]. It is active against Gram-positive organisms, with little activity against enteric Gram-negative bacilli [19]. In 2009, the U.S. Food and Drug Administration prevented use of novobiocin capsules on the market because of adverse reactions including relatively common skin reactions, jaundice, hepatic failure, and blood dyscrasias, as well as concerns about the development of novobiocin-resistant staphylococci during treatment and potential for drug interactions [20]. However, novobiocin is still used in veterinary as feed supplement for chickens and turkeys (U.S. Food and Drug Administration Code of Federal Regulations 21CFR558.415, Revised as of April 1, 2014), and topically for treatment of bovine mastitis in lactating cattle (licensed by the UK Veterinary Medicine Directorate). An alternative aminocoumarin, clorobiocin, had increased efficacy compared to novobiocin against Bcc 
bacteria and also enhanced polymyxin B activity. Greater clorobiocin efficacy may be the result of stronger enzyme inhibition by clorobiocin as previously shown against the DNA gyrase and topoisomerase IV proteins from both E. coli and Staphylococcus aureus [21]. Alternatively, differences between novobiocin and clorobiocin might be due to other clorobiocin targets, for example clorobiocin can inhibit the phosphoserine phosphatase SerB2 of Mycobacterium tuberculosis while novobiocin cannot [22].

The observation that novobiocin and clorobiocin at low concentrations synergize with $\mathrm{PmB}$ and colistin against Bcc isolates suggests that these combinations could be considered for treatment of these pan-antibiotic resistant bacteria. Recently, Moore et al. showed that novobiocin displayed potent antimicrobial activity against $20 \mathrm{~B}$. cenocepacia $\mathrm{CF}$ isolates that are irresponsive to any other antibiotic [28]. In that study, $30 \mathrm{P}$. aeruginosa CF isolates were not affected by novobiocin. These observations agree with our data concerning the susceptibility of the different bacterial species to novobiocin. Safety concerns resulted in withdrawal of human use of novobiocin [20]; however, novel drug delivery systems based on a nebulized solution or a dry powder inhaler that would deliver enhanced concentrations of aminocoumarins locally could control systemic toxicity and treat Bcc infections [28]. We further suggest their use in combination with colistin due to the synergistic effects of both antibiotic classes against Bcc bacteria. This will also facilitate using lower doses of each antibiotic, reducing systemic toxicity. In addition, using aminocoumarin derivatives with enhanced potency against Bcc bacteria such as clorobiocin would allow further lowering the aminocoumarin concentration in the antibiotic combination. In conclusion, the results of this study, in addition to recent supporting evidence [28], warrant reconsideration of novobiocin, clorobiocin or other aminocoumarins for human use in combination with colistin administered by a novel delivery system to control infections by multidrug resistant Bcc bacteria. There are reports in the literature of select antimicrobial peptides with increased activity against Burkholderia sp. [29, 30] and the efficacy of these compounds in conjunction with an aminocoumarin would also be worth exploring.

\section{Acknowledgements}

The authors thank Ivan Villanueva for excellent technical support and Dr. Elizabeth Tullis for Bcc isolates GIB-C1141144 and GRI-C1210932. This work was supported by grants from the Canadian Institutes of Health Research Special Initiative on Novel Antimicrobials and the Seventh Framework Programme Marie Curie Career Integration grant PCIG13-GA2013-618095 (to M.A.V.), and Cystic Fibrosis Canada (to D.P.S.). M.A.V. held a Canada Research Chair in Infectious Diseases and Microbial Pathogenesis. O.M.E. was supported by an Ontario Graduate Scholarship. S.A.L. was supported by a Cystic Fibrosis Canada studentship and an NSERC postdoctoral fellowship. A.N.J. was supported by a Michael Smith Foundation studentship. 
Table 1: FIC indices for combinations of novobiocin with other antibiotics

\begin{tabular}{llll}
\hline \multirow{2}{*}{ Species/strain } & \multicolumn{3}{c}{ FIC index for novobiocin +} \\
\cline { 2 - 4 } & PmB & Colistin & Tobramycin \\
\hline B. cenocepacia K56-2 & 0.31 & 0.28 & 0.63 \\
\hline B. multivorans C0514 & 0.28 & 0.5 & 0.63 \\
\hline B. cenocepacia GIB-C1141144 & 0.5 & 0.56 & $>1.0$ \\
\hline B. cenocepacia GRI-C1210932 & 0.31 & 0.38 & $>1.0$ \\
\hline
\end{tabular}

Table 2: Novobiocin and clorobiocin MIC distribution amongst Bcc bacteria

\begin{tabular}{|c|c|c|}
\hline \multirow{2}{*}{$\begin{array}{l}\mathrm{MIC} \\
(\mu \mathrm{g} / \mathrm{ml})\end{array}$} & \multicolumn{2}{|c|}{ Bcc isolates (30) } \\
\hline & Novobiocin & Clorobiocin \\
\hline 1 & 0 & 1 \\
\hline 2 & 1 & 0 \\
\hline 4 & 0 & 14 \\
\hline 8 & 0 & 12 \\
\hline 16 & 2 & 3 \\
\hline 32 & 12 & 0 \\
\hline 64 & 15 & 0 \\
\hline
\end{tabular}




\section{Legend to Figures}

Figure 1. Five compounds from the library of 2686 compounds showed synergistic effects with $500 \mu \mathrm{g} / \mathrm{ml} \mathrm{PmB}$ against B. cenocepacia K56-2. Results are shown as percentage inhibition of the growth in the presence of compounds relative to that in the absence of compounds whether alone (X-axis) or in combination with PmB (Y-axis). A black circle represents each compound. The red circle represents the results of all wells with no library compound added (1 well per plate). Compounds considered significantly inhibitory on their own or synergistic with PmB had to inhibit growth by more than $70 \%$ (dashed lines with shaded areas representing higher levels of inhibition). The dotted line has a slope of one and intersects with the origin; it is drawn for illustrative purposes only. Points in the upper left quadrant represent compounds that do not directly inhibit $B$. cenocepacia growth at the concentration tested but that synergize with PmB. Points in the lower right quadrant represent compounds that inhibit B. cenocepacia but are antagonized by PmB. Points near the line in the upper right quadrant represent compounds that inhibit B. cenocepacia but that do not increase or decrease the effect of PmB. The compounds are: A, IKD-8344; B, Novobiocin; C, CL0231; D, Micacocidin B; E, Thielavin B.

Figure 2. Chemical structures of compounds discussed in the text. A, Thielavin B. B, Micacocidin B. C, IKD-8344. D, CL0231. E, CL0236. F, Novobiocin. G, Clorobiocin. Structures were drawn using Marvin Sketch Version 14.12.8. 


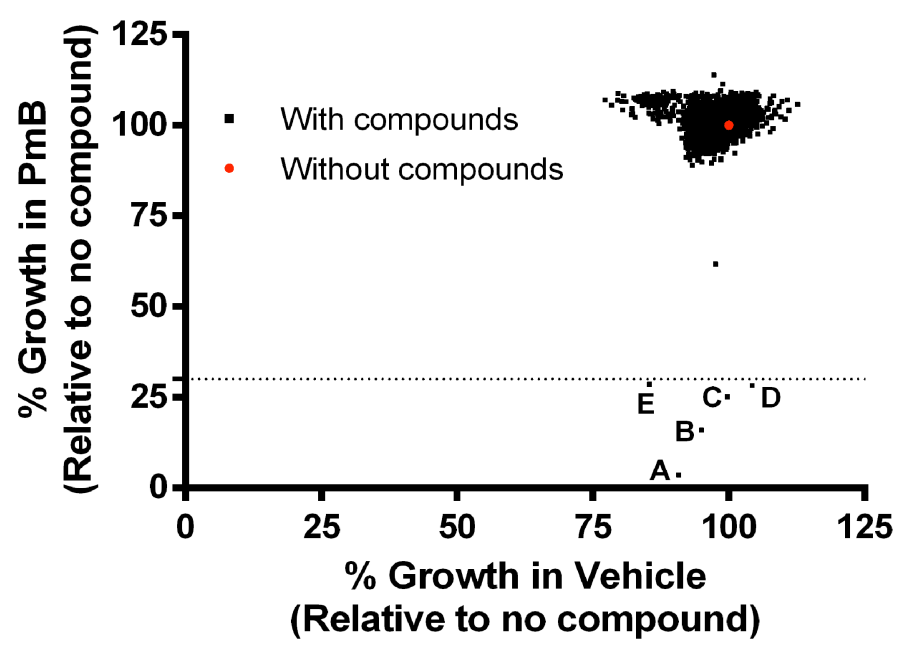

Figure 1 


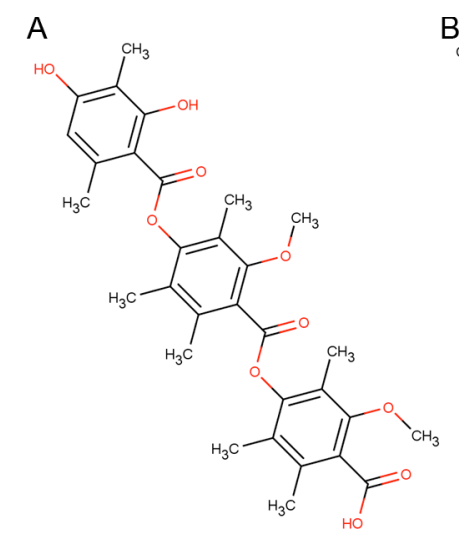

D

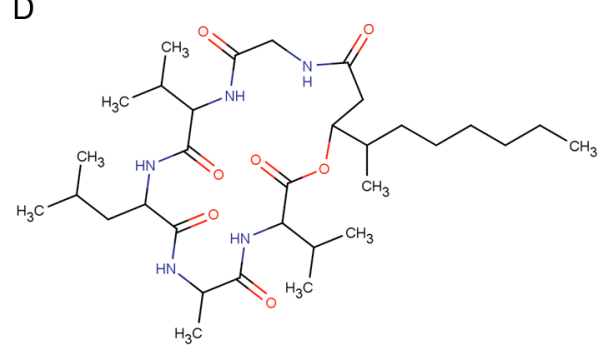

F

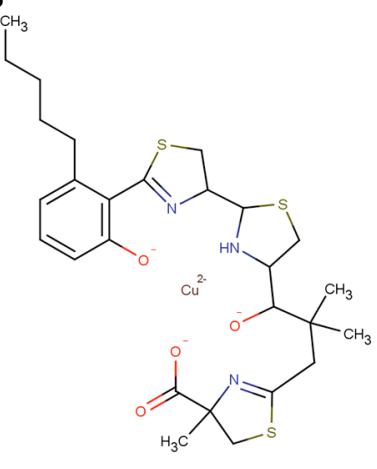

B C

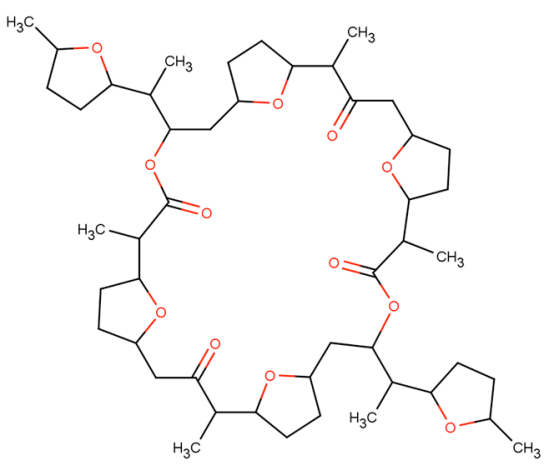

E

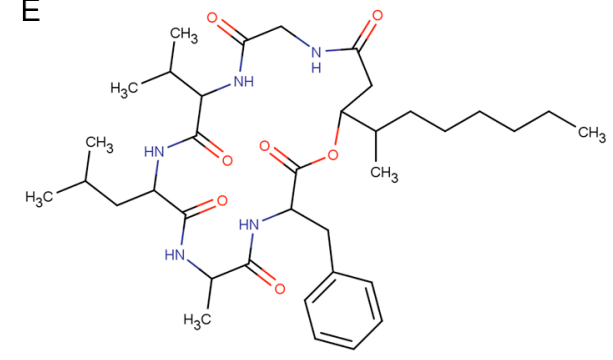

G

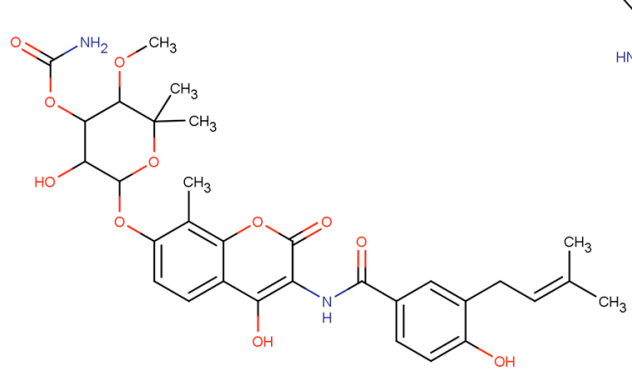

Figure 2 


\section{References}

[1] WHO-Media-Centre. The 10 leading causes of death by broad income group, 2004. Fact sheets: World Health Organization; 2008.

[2] Waters V. New treatments for emerging cystic fibrosis pathogens other than Pseudomonas. Curr Pharm Des 2012;18:696-725.

[3] Biswas S, Brunel JM, Dubus JC, Reynaud-Gaubert M, Rolain JM. Colistin: an update on the antibiotic of the 21st century. Expert Rev Anti Infect Ther 2012;10:917-34.

[4] Loutet SA, Valvano MA. Extreme antimicrobial peptide and polymyxin B resistance in the genus Burkholderia. Front Microbiol Cell Inf Microbiol 2011;2:159.

[5] Loutet SA, Valvano MA. A decade of Burkholderia cenocepacia virulence determinant research. Infect Immun 2010;78:4088-100.

[6] Speert DP, Henry D, Vandamme P, Corey M, Mahenthiralingam E. Epidemiology of Burkholderia cepacia complex in patients with cystic fibrosis, Canada. Emerg Infect Dis 2002;8:181-7.

[7] De Soyza A, Ellis CD, Khan CM, Corris PA, Demarco de Hormaeche R. Burkholderia cenocepacia lipopolysaccharide, lipid A, and proinflammatory activity. Am J Respir Crit Care Med 2004;170:70-7.

[8] Wright GD, Sutherland AD. New strategies for combating multidrug-resistant bacteria. Trends Mol Med 2007;13:260-7.

[9] El-Nakeeb MA, Abou-Shleib HM, Khalil AM, Omar HG, El-Halfawy OM. Reversal of antibiotic resistance in Gram-positive bacteria by the antihistaminic azelastine. Apmis 2012;120:215-20.

[10] Mahenthiralingam E, Coenye T, Chung JW, Speert DP, Govan JR, Taylor P, et al. Diagnostically and experimentally useful panel of strains from the Burkholderia cepacia complex. J Clin Microbiol 2000;38:910-3.

[11] Loutet SA, Bartholdson SJ, Govan JR, Campopiano DJ, Valvano MA. Contributions of two UDP-glucose dehydrogenases to viability and polymyxin B resistance of Burkholderia cenocepacia. Microbiology 2009;155:2029-39.

[12] Vaara M, Porro M. Group of peptides that act synergistically with hydrophobic antibiotics against Gram-negative enteric bacteria. Antimicrob Agents Chemother 1996;40:1801-5.

[13] Jassem AN, Zlosnik JE, Henry DA, Hancock RE, Ernst RK, Speert DP. In vitro susceptibility of Burkholderia vietnamiensis to aminoglycosides. Antimicrob Agents Chemother 2011;55:2256-64.

[14] Kitahara N, Endo A, Furuya K, Takahashi S. Thielavin A and B, new inhibitors of prostaglandin biosynthesis produced by Thielavia terricola. J Antibiot 1981;34:1562-8.

[15] Mani N, Sancheti P, Jiang ZD, McNaney C, DeCenzo M, Knight B, et al. Screening systems for detecting inhibitors of cell wall transglycosylation in Enterococcus. Cell wall transglycosylation inhibitors in Enterococcus. J Antibiot 1998;51:471-9.

[16] Kobayashi S, Hidaka S, Kawamura Y, Ozaki M, Hayase Y. Micacocidin A, B and C, novel antimycoplasma agents from Pseudomonas sp. I. Taxonomy, fermentation, isolation, physicochemical properties and biological activities. J Antibiot 1998;51:323-7.

[17] Ishida T, In Y, Nishii M, Minami Y. Stereochemistry of a novel macrodiolide antibiotic IKD-834. Chem Lett 1994;23:1321-2.

[18] Maxwell A. The interaction between coumarin drugs and DNA gyrase. Mol Microbiol 1993;9:681-6. 
[19] Greenwood D. Synthetic antibacterial agents and miscellaneous antibiotics. In: Greenwood D, editor. Antimicrobial Chemotherapy. 4th ed. Oxford: Oxford University Press; 2000. p. 4658.

[20] https://federalregister.gov/a/2011-1000. Accessed: February 13, 2015.

[21] Alt S, Mitchenall LA, Maxwell A, Heide L. Inhibition of DNA gyrase and DNA topoisomerase IV of Staphylococcus aureus and Escherichia coli by aminocoumarin antibiotics. J Antimicrob Chemother 2011;66:2061-9.

[22] Arora G, Tiwari P, Mandal RS, Gupta A, Sharma D, Saha S, et al. High throughput screen identifies small molecule inhibitors specific for Mycobacterium tuberculosis phosphoserine phosphatase. J Biol Chem 2014;289:25149-65.

[23] De Leon GP, Elowe NH, Koteva KP, Valvano MA, Wright GD. An in vitro screen of bacterial lipopolysaccharide biosynthetic enzymes identifies an inhibitor of ADP-heptose biosynthesis. Chem Biol 2006;13:437-41.

[24] Kline T, Trent MS, Stead CM, Lee MS, Sousa MC, Felise HB, et al. Synthesis of and evaluation of lipid A modification by 4-substituted 4-deoxy arabinose analogs as potential inhibitors of bacterial polymyxin resistance. Bioorg Med Chem Lett 2008;18:1507-10.

[25] Hamad MA, Di Lorenzo F, Molinaro A, Valvano MA. Aminoarabinose is essential for lipopolysaccharide export and intrinsic antimicrobial peptide resistance in Burkholderia cenocepacia. Mol Microbiol 2012;85:962-74.

[26] Loutet SA, Flannagan RS, Kooi C, Sokol PA, Valvano MA. A complete lipopolysaccharide inner core oligosaccharide is required for resistance of Burkholderia cenocepacia to antimicrobial peptides and bacterial survival in vivo. J Bacteriol 2006;188:2073-80.

[27] El-Halfawy OM, Valvano MA. Chemical communication of antibiotic resistance by a highly resistant subpopulation of bacterial cells. PLOS One 2013;8:e68874.

[28] Moore JE, Alcorn M, Ara W, Millar BC, McCaughan J, Rendall JC, et al. Do veterinary antibiotics have efficacy against highly resistant Gram-negative pathogens from patients with cystic fibrosis? Int J Antimicrob Agents 2015;45:93-5.

[29] Thwaite JE, Humphrey S, Fox MA, Savage VL, Laws TR, Ulaeto DO, et al. The cationic peptide magainin II is antimicrobial for Burkholderia cepacia-complex strains. J Med Microbiol 2009;58:923-9.

[30] Samy RP, Thwin MM, Stiles BG, Satyanarayana-Jois S, Chinnathambi A, Zayed ME, et al. Novel phospholipase A2 inhibitors from python serum are potent peptide antibiotics. Biochimie 2015;111:30-44. 